\title{
Selection of Temporally Dithered Codes for Increasing Virtual Depth of Field in Structured Light Systems
}

\author{
Ricardo R. Garcia \\ UC Berkeley \\ Berkeley, CA \\ rrgarcia@eecs.berkeley.edu
}

\author{
Avideh Zakhor \\ UC Berkeley \\ Berkeley, CA \\ avz@eecs.berkeley.edu
}

\begin{abstract}
Temporally dithered codes have recently been used for depth reconstruction of fast dynamic scenes using off-theshelf DLP Projectors. Even though temporally dithered codes overcome the DLP projector's limited frame rate, limitations with the optics create challenges for using these codes in an actual structured light system. Specifically, to maximize the amount of light leaving the projector, projector lenses are designed to have large apertures resulting in projected patterns that appear in focus over a narrow depth of field. In this paper, we propose a method to design temporally dithered codes in order to extend the virtual depth of field of a structured light system. By simulating the PWM sequences of a DLP projector and the blurring process from the projector lens, we develop algorithms for designing and decoding projection patterns in the presence of out of focus blur. Our simulation results show a 47\% improvement in the depth of field when compared against randomly selected codewords.
\end{abstract}

\section{Introduction}

There has been a great deal of interest in developing tools and methodologies for capturing 3D depth models of scenes for many years [1]. These depth capture methods can be generally classified as either passive or active. The most common passive and active methods are stereo vision and structured light, respectively [1].

In general, structured light systems have been developed around off-the-shelf digital projectors with limited modifications [2, 3, 4, 5, 6]. Since these projectors are not designed for structured light applications, some of their inherent qualities become problematic for depth capture. The most significant of these problems is due to the projector lenses, which are generally selected with large apertures to maximize the amount of light leaving the projector [4]. Unfortunately, increasing the aperture of a lens reduces the depth of field (DOF) of the projector. The patterns projected onto the scene only appear in focus if they fall onto objects whose distance to the projector is close to the distance at which the projector is focused [7].

Most patterns used in structured light systems are composed of multiple sequentially projected frames [1]. In order to determine the projected pattern at each position, the scene must be static while each frame of the pattern is projected. Since most DLP projectors are only capable of projecting patterns at a rate of 60 frames per second (fps), it is difficult to use these projectors to capture fast moving dynamic scenes. Temporally dithered codes have recently been shown to significantly increase the rate at which codes are projected from a standard DLP projector [2]. These codes make it possible to capture dynamic scenes with faster motion than has been possible with traditional 60 fps projection.

In this paper, we present a systematic way to select temporally dithered codes that increase the virtual DOF of a structured light system of the kind presented in [2]. We refer to this as an increase in "virtual" DOF in order to distinguish it from other existing approaches in which the DOF is increased in a physical way by changing actual hardware configurations of the projector or the camera [4, $8,9,10,11]$. Our basic approach is to simulate temporally dithered patterns in order to choose codes that (a) are maximally separated when in focus in order to be resilient to noise, and (b) handle high frequency attenuation due to out of focus blurring. The patterns are generated by simulating the PWM operation of the DLP projector. We use basic lens geometry to relate our blurring models to the DOF of a system. By increasing the tolerable size of blur, represented by the standard deviation of a Gaussian function, we increase the virtual DOF of the system. The relationship between improvements in tolerable blur size and DOF improvements are dependent on system parameters. We provide several examples of the potential virtual DOF improvements gained by our proposed method.

The outline of the paper is as follows: in Section 2, we provide a background of structured light systems and depth of field improvement. Section 3 describes our simulation setup; Section 4 presents methods for code selection. Section 5 presents the idea of "depth codes" for improving the virtual DOF of a structured light system. 
Section 6 provides results on how the improvements in blur tolerance affect the DOF of an actual projector. Discussion and future work are presented in Section 7.

\section{Background}

A significant amount of effort has been focused on designing patterns for structured light systems. In [1], a comprehensive overview of the different coding strategies is presented. The basic goal is to find a set of codes that is able to uniquely identify spatial regions in the projection pattern by changing intensities or colors over time and space. A common choice in structured light systems is to assign a unique code to each column of pixels in the projector. These codes are usually made up of multiple consecutively displayed intensities. To encode the scene with these codes, a sequence of image frames is projected, where each column displays the corresponding sequence of intensities from its assigned code. The set of consecutive image frames is referred to as the projection pattern.

Digital projectors, especially DLP projectors, have been used extensively in structured light systems [2, 3, 5, 12, 13]. Even though DLP projectors are capable of producing images comparable in color quality and speed to other projection technologies, their principles of operation offer additional flexibility as compared to others. The DLP projector's speed of operation makes it particularly well suited for structured light systems. For example, Zhang et. al. [3] encode the three frames used in their sinusoidal projection pattern in the RGB color components of a single video frame. Each of the three sinusoidal patterns is projected sequentially as the projector displays RGB components of the image.

Blurring in optical systems that have a limited depth of field is a well understood phenomenon. In many systems, the blurring from a limited depth of field can make the desired imaging goals difficult to accomplish [6]. In projector systems, a limited depth of field means that a projected image only appears in focus if it falls on a surface near the distance of focus. Attempts have been made to reduce the effects of this blurring through modified optics and signal processing techniques $[4,6,8$, $9,11,14]$. In [6], a method is presented to separate global illumination and out of focus blurring in a structured light system. By measuring the blur present throughout the scene, a reconstruction of the scene is possible, even in the presence of global illumination. In [9], Levin et. al. replace the aperture in a camera lens with a coded aperture. With this modified aperture, the effects from blurring can be minimized after capture by filtering with the inverse of the point spread function. In [14], Grosse and Bimber make a similar modification to the aperture of a projector. With the modified aperture, the projected pattern can be pre-filtered to compensate for the out of focus blur. In [4], Bimber and Emmerling increase the depth of field of a structured light system by merging the projections from multiple digital projectors focused at different depths. Although these methods do increase the depth of field of optical systems, they require additional hardware and real-time data processing that makes their implementation difficult in structured light systems capturing dynamic scenes.

Narasimhan et. al. proposed a method to significantly increase the number of patterns that could be captured from a DLP projector in a single frame lasting $1 / 60^{\text {th }}$ of a second [2]. Rather than capturing patterns in the color channels, they capture the pulse width modulated PWM patterns at a very high rate. These captured patterns are different for different RGB values, and can be used as unique codes corresponding to a temporal dithering of the modulating pixels. Even though this temporal dithering increases the rate at which structured light patterns are projected, there are no existing schemes for choosing patterns for projection. In this paper, we propose a number of ways to design temporally dithered codes for a system of the kind in [2].

\section{Simulation Setup}

We choose to use realistic parameters for the simulation of the cameras and projectors in the system. The properties of the projector are modeled after an Optoma TX 780, which operates at $60 \mathrm{~Hz}$, and has a 5-segment color wheel which rotates at $2 \times$ the $60 \mathrm{~Hz}$ frame rate. We assume the color wheel has been removed so that the projector operates in grayscale. The projector's lens has a $28 \mathrm{~mm}$ focal length with an f-number of 2.5.

As for the capture process, we choose the capture parameters based on a Point Grey Dragonfly Express camera with 10 bits of dynamic range. At its highest capture speed, the camera is capable of capturing $200 \mathrm{fps}$. The camera should capture an integer number of frames during a single video frame. Therefore, our simulation assumes that the camera operates at $180 \mathrm{fps}$, giving each dithered code a set of three intensities. In our simulation, we do not account for potential blurring that could occur from capturing the projected pattern with a camera. We choose a capture resolution equal to that of our projected pattern. We assume a projection width of 1024 pixels. As such, our goal is to design a set of 1024 temporally dithered codes.

\subsection{Modeling Blur}

The DOF in traditional camera optics refers to the range of distances over which a single point is projected onto the image to an area with a size smaller than a specified radius. This tolerable radius of blur is referred to as the circle of confusion. In our context, the DOF of a 
structured light system is defined as the range of distances over which the corresponding circle of confusion is the largest blur size tolerated before the projection patterns can no longer be correctly decoded. Using simple geometric calculations we can relate the radius of blur to the distance the object is from the plane of focus, as shown in Figure 1.

In Figure 1, let $r_{0}$ be the size of the lens' aperture, and $r_{b}$ be the radius of the tolerable circle of confusion. Let $d$ be the distance between the lens and projector image plane if the projector is focused at $D$, and let $d_{F}$ be the distance that a point at $D_{F}$ would appear in focus, but would have a circle of confusion of radius $r_{b}$ at distance $d$. Simple geometry in Figure 1 enables us to solve for the size of the circle of confusion:

$$
r_{b}=r_{0}\left(\frac{d}{d_{F}}-1\right)
$$

Given the maximum tolerable size of blur $r_{b}$, the focal length of the lens $f$, the f-number of the lens $N$, and the distance $D$ to the focused plane, we can solve for the DOF of the system [7]:

$$
D O F=\frac{2 d f^{2} N(D-f) r_{b}}{f^{4}-r_{b}^{2}(N(D-f))^{2}}
$$

In an actual optical system, the blur due to defocus is not a true circle. Rather, it has a circularly symmetric shape with a soft edge that is caused by a combination of aberration from the lens and diffraction from the aperture. This shape is generally modeled as a two-dimensional Gaussian function. The radius of blur is related to the standard deviation of the Gaussian function as [15]:

$$
\sigma=\rho r_{b}
$$

The parameter $\rho$ is dependent on the aberration from the lens as well as the diffraction from the aperture and is set to $\rho=1$ in our simulations. In this paper we do not take into account changes in light intensity due to changes in distance of objects. Specifically, we assume that the change in intensity is minimal over the range of distances of interest.

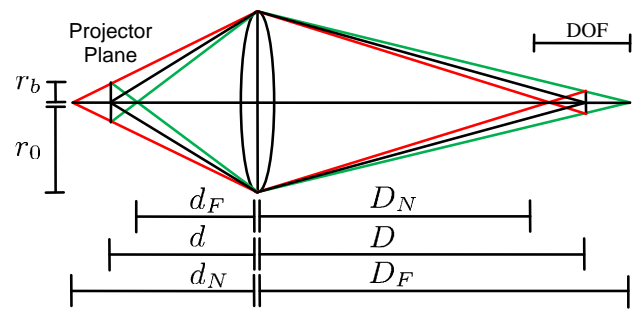

Figure 1: Geometry of circle of confusion in simple lens.

\subsection{Simulating PWM Patterns}

In order to design patterns that are robust to blurring, we must simulate the temporally dithered patterns. Unfortunately, the exact PWM patterns used in DLP projectors are proprietary and not available in public domain. Despite this, several known key properties of the DLP operation allow reasonable patterns to be simulated. Figure 2 illustrates a simple method to generate a PWM sequence from a binary number.

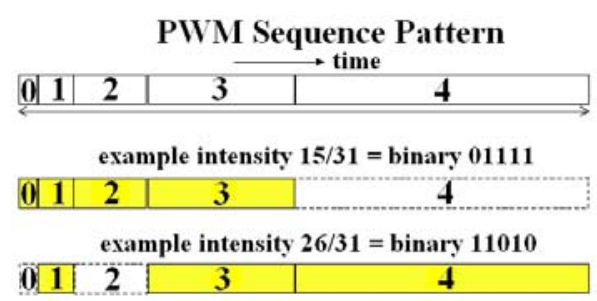

Figure 2: Simple mapping of a binary number to PWM sequence. The values 0 through 4 represent each of the bits in the binary number [16].

The relative duration of the $\mathrm{K}^{\text {th }}$ bit in the PWM sequence is $2^{\mathrm{K}} /\left(2^{\mathrm{M}}-1\right)$, where $\mathrm{M}$ is the number of bits in the binary number. In the PWM sequences used by DLP projectors, the portions of the sequence that correspond to higher order bits are divided in smaller sections rather than being displayed in a single contiguous segment. This is done to create more visually appealing images [16]. For simulation purposes, we use a "bit-splitting" scheme, shown in Figure 3, to create the PWM pattern [16]. This method is closer to PWM sequences used by DLP projectors than the simple method shown in Figure 2. The ordering of the divided segments in Figure 3 follows a basic pattern: a segment for the most significant bit is placed in every other segment of the PWM sequence. The next most significant bit is placed once every four segments. The pattern is continued in this way until the two least significant bits are placed in only a single location. Note that the duration of the least significant bit is half that of all other bit segments.

\subsection{Choosing Color Codes}

For our simulations, we assume that the projector operates with the color wheel removed. Without the color wheel, each of the RGB color channels projects as white light with varying grayscale intensities. By projecting only white light and carefully choosing RGB color combinations, it is possible to ensure that the chosen patterns are not visible to the naked eye. For example, in structured light systems using sinusoidal fringe patterns, as in [3], the rapid sequential projection of the three phase shifted patterns results in a scene that appears to be illuminated by a constant light source. This is ideal for 
capturing the underlying texture of the scene with a separate camera during the reconstruction process. With a camera capturing at $60 \mathrm{fps}$, each of the fast varying patterns is integrated and captured at a constant intensity. We can determine color codes with the same intensity in the absence of the color wheel by calculating how long light from each of the color channels is projected. Since the projector uses 24-bit color, it is possible to iterate through all 16,777,216 color combinations to determine the resulting intensity of each color code.

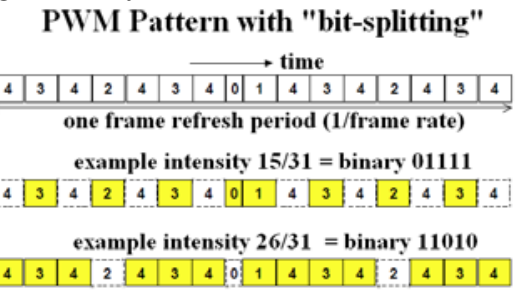

Figure 3: PWM sequence using "bit-splitting" method. The higher order bits are split into multiple positions to create more visually appealing images from the DLP projector [16].

The 5-segment color wheel in the Optoma TX780 projector uses the following color sequence for each video frame: red, yellow, green, blue, white, red, yellow, green, blue, and white. In our simulations, we assume that no light is projected during the white and yellow color segments. In practice, this can be accomplished by operating the projector in "photographic" mode. Even though the yellow and white segments can potentially add more unique information when capturing the temporally dithered codes, the unknown structure of the derived color channels makes any attempts to simulate the temporally dithered color codes inaccurate. Figure 4 shows the timing of how the camera captures the PWM sequences within each of the projector's video frames.

To find blur resilient codes, we first compute the intensities of all possible RGB values. To minimally affect the texture capture and reconstruction and minimize the affect of projected patterns to the naked eye, we limit our codes to RGB values resulting in constant intensity. Furthermore, it is desirable to choose a constant intensity level whose codes take on a large range of values across each of the three components of a code. For example, selecting a very dark (or bright) intensity results in all of the corresponding codes consisting of all three components near 0 (or 255). By choosing an intensity closer to the middle of the range, e.g. 128, the three components of the code are more likely to span a large range of values, thus taking advantage of the dynamic range of the capturing camera. Another consideration is to choose a constant intensity level with a large set of associated codewords. The larger the pool of available codewords, the more degrees of freedom there are in the subsequent set for choosing blur resistant codes. Based on these considerations, we opt to use RGB color codes with a constant intensity of 147 out of the maximum brightness of 255 for the simulations in this paper. As it turns out, intensity value of 147 results in the greatest number of codewords out of 16 million, i.e. 400,000. Furthermore, the variation of intensities across the codes is large. Even though we choose 147 as our intensity, our approach in this paper is applicable to other intensity values.

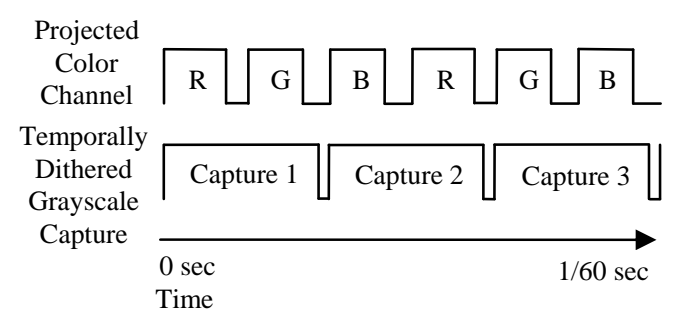

Figure 4: Timing diagram of projector color channels, temporally dithered grayscale code capture at $180 \mathrm{~Hz}$.

\subsection{Creating Captured Patterns}

For each RGB color code, we simulate the camera's light integration of the corresponding PWM sequences during a single video frame. Figure 3 shows a sequence of segments representing a PWM pattern. Starting with the red PWM sequence, if the first segment has a one, we increase a running sum that represents the amount of captured light in the current image capture. If the first segment is a zero, the running sum is unchanged. For each new segment, we subtract the segment duration from the time remaining in the current capture. We increment through the entire PWM sequence until we either reach the end of the PWM sequence or run out of time on the current capture. If we reach the end of a color channel's PWM sequence, we subtract the time interval before the next PWM sequence and continue with the next color's PWM sequence. If we reach the white or yellow segments, we simply subtract their duration from the remaining time on the current capture since we do not expect the projector to be on during these periods. Once we have captured all of the color codes, we normalize the results such that the brightest pattern has a value equal to the brightest pixel in the camera. In this work, we assume that we can calibrate the camera such that we can use the full 10-bit dynamic range of the imaging sensor.

\section{Code Selection}

One of the main challenges in our simulations is the choice of color codes within the projected pattern. Specifically, we have to choose among the 400,000 color codes corresponding to a desired intensity of 147 . For all of the possible color codes, the corresponding dithered codes are determined and used to select a final set of 
codes. The key challenge is to not only choose the "right" codes, but also to correctly position them in the overall projected pattern. Since codes are assigned to entire columns in the projected pattern, we only need to determine a code ordering from left to right.

Selecting the proper subset of codes to use in a projected pattern requires several criteria to be met. Firstly, it is desirable to choose a set of codes that minimizes the probability of decoding error under focused conditions. Secondly, the selected codewords must remain identifiable under blurring.

\subsection{Maximally Separated Codes}

In order to accurately detect which code is projected onto each point in the scene, each of the chosen codes needs to be easily distinguishable from the other projected codes. When performing vector detection under the assumption of additive noise, we use maximum likelihood evaluations to decode in order to minimize the probability of decoding error [17]. Let us assume the observation vector $y$ is generated by:

$$
y=x+w
$$

where $x$ is the projected codeword and $w$ is the noise in the observation. In our simulation, $x \in S_{1024}$, where $S_{1024}$ is the set of 1024 codes we project. To decode, we find the maximum likelihood solution by evaluating:

$$
\arg \max _{x \in S_{1024}} P(y \mid x)
$$

In the case of additive Gaussian noise, the maximum likelihood detector reduces to choosing the code closest to our observation vector [17]:

$$
\min _{x \in S_{1024}}\|y-x\|^{2}
$$

Errors in decoding occur when noise pushes the observation such that it is closer to an incorrect code than the correct code. In choosing our codes, it is desirable reduce the probability that incorrect detections occur. To minimize the probability of error for a given noise level, we must increase the distance between all of the possible symbols. This can easily be visualized in the one dimensional case, as shown in Figure 5. Suppose we are detecting a binary symbol, A or B, in the presence of Gaussian noise. Figure 5 shows the distribution of possible observed values. The light gray shaded areas indicate the probability of erroneous decoding using the maximum likelihood detection method. With the given noise distribution, it is clear that if we increase the distance between the two possible codes, to $\mathrm{A}^{\prime}$ and $\mathrm{B}$ ', we decrease the probability of error in decoding, represented by the red shaded area. Although separating the codes does reduce the probability of error, there are limitations as to how much separation can be applied. In a structured light system, we are limited to the dynamic range of the projector which produces intensities from 0 - 255. This limitation is similar to the transmit power constraint in communication systems.

Code separation can be generalized to the multidimensional vector case. Viewing the potential set of codewords in three dimensional space, we see that the set of codewords with constant intensity of 147 all lie on a single plane:

$$
x_{1}+x_{2}+x_{3}=147
$$

which is located in the first octant of the three dimensional space. If our projector could generate the set of all points on this plane, it would be trivial to find maximally separated points. However, since in practice this is not the case, we must devise a selection method to guarantee that points chosen on this plane are well separated. To maximize resilience against additive noise and minimize the probability of error during the codeword decoding process, our first criterion for selecting a set of codewords is to maximize the distance between all codewords in the set.

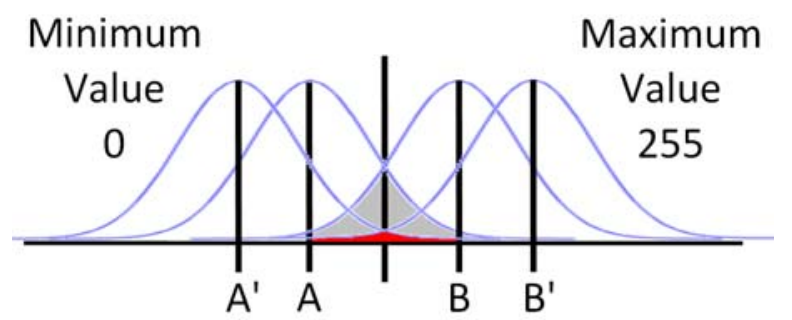

Figure 5. Binary Dection under noise.

To achieve this, we propose an iterative selection method: the first code is chosen randomly from the available set of codes. To choose subsequent codes, we evaluate the distance of each candidate code to each of the already selected codes. We store the minimum of these distances and perform the same evaluation on the remaining candidate codes, also storing their minimum distances. When all of the candidate codes have been evaluated, we choose the candidate code with the maximum minimum distance. Even though the resulting set of codewords is dependent on the initially chosen codeword; due to computational reasons, we do not optimize over this codeword.

This maximization of the minimum neighbor distance ensures that the selected codewords are well separated from each other and are thus robust to noise during the decoding process. We refer to this non-ordered, maximally separated code set as $C_{M S}$.

\subsection{Ordering Selected Codes}

The blurring function in standard optics is a spatial lowpass filter. Examining the spectrum of a focused and out of focus version of an image, we note that the majority of 
the lost signal energy is from the high frequency content of the image. Therefore, to make our projected pattern resilient under blurring conditions, it is desirable to reduce the high frequency content that is present in the projected pattern. This can be accomplished through the careful ordering of codes within the projection pattern.

The code selection method in Section 4.1 allows us to arrive at a set of codes that is robust to noise in focused conditions. However, this method does not take into account any interactions between spatially neighboring codes when blurring occurs. For a projected pattern with significant energy at high spatial frequencies, blurring causes much of the unique projected information to be lost. In contrast, if the pattern contains most of its energy at low spatial frequencies, it suffers from less distortion under blurring, and thus the projected codes are easier to detect.

It is clear that a signal with low frequency content changes its value slower compared to a signal with high frequency content. Exploiting this, we choose to reduce the high frequency content of the projected pattern by ensuring that neighboring codewords are near each other in the Euclidean space. At first glance, this might appear to be in contradiction with the previous process where we maximized the distance between all the words in the code set. Indeed, there is no contradiction. In general detection problems, it is advantageous to choose symbols to be far apart from each other [17]. However, once the symbol set is chosen in a maximally separated manner, they should be spatially arranged in a smooth way so that their frequency content is not attenuated after blurring.

To achieve a spatially smooth code set ordering, we position the first code in our set $C_{M S}$ at the left most position in the pattern and then search for the closest remaining code in $C_{M S}$ to place in the position next to it. As we iterate through each position, we search for the closest code out of the remaining codes to place next to it, i.e. to its right. Thus, we rearrange $C_{M S}$ from left to right in a spatially smooth manner. We refer to the resulting code set as $C_{M S}^{L F}$, where $L F$ stands for low frequency. This method results in a projected pattern with significantly smaller high spatial frequency content as compared to the non-ordered $C_{M S}$.

Figures 6 and 7 show the set of intensities making up the first frame of $C_{M S}$ and $C_{M S}^{L F}$ respectively. The solid blue lines in both these figures contain the same set of values corresponding to frame 1 intensity capture values for both schemes. As seen, the ordering of the codewords in Figure 7 obviously results in a signal with much lower spatial frequency content.

\subsection{Performance of Codes}

We now need to determine the amount of blur the codes can tolerate before they are no longer decodable. To do so, we model the blur from the optics as a Gaussian function, and convolve the columns of the pattern with this Gaussian function. Since the intensities down each column of pixels are constant, we perform our blurring using a one dimensional Gaussian function across the columns of the projected pattern even though in practice blurring is a two dimensional process. The captured codes are then quantized and decoded with respect to the original codes. The decoding process computes the Euclidean distance between the blurred codewords and the original code set. Error-free decoding is achieved if each blurred codeword is closest to the focus codeword in the same position.

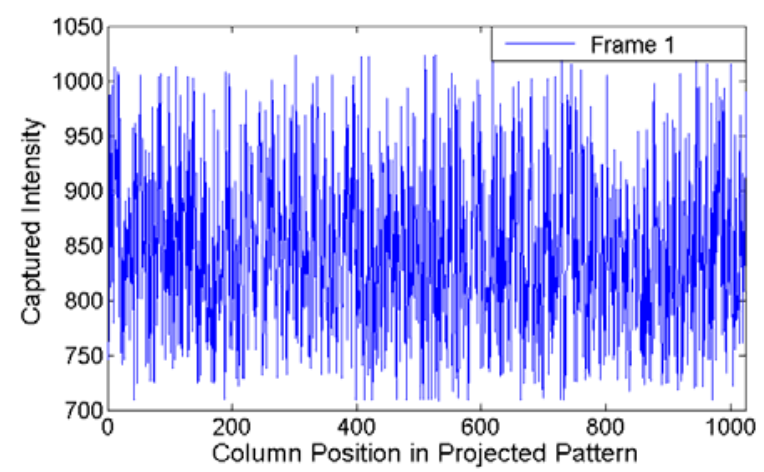

Figure 6: Intensity values for frame 1 of $C_{M S}$.

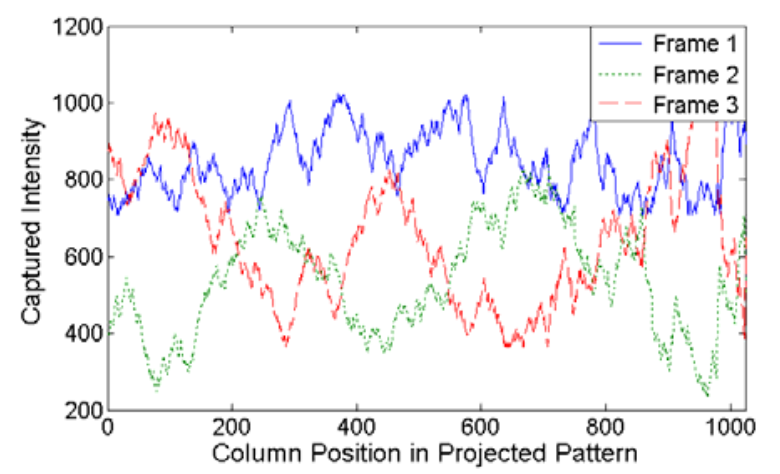

Figure 7: Intensity values for $C_{M S}^{L F}$. All three frames for $180 \mathrm{~Hz}$ codes are shown.

To determine the Maximum Tolerable Blur (MTB), we decode projection patterns blurred with an increasingly wider Gaussian function until decoding becomes erroneous. To define the Gaussian function, we choose a blur increment value, $\Delta \sigma_{b}$. Since we are blurring discrete codes, we create a discretized Gaussian function with width parameter $\sigma_{b}=\Delta \sigma_{b} I_{B}$, where $I_{B}$ is the blur index and $\Delta \sigma_{b}=0.001$. We quantify our decoding results in terms of the MTB index. 
To verify that maximally separated and ordered codewords result in better performance under out of focus blurring, approximately 1000 random sets of 1024 codes are chosen out of the 400,000 possible dithered codes, and their average MTB size is found to be at blur index 283. We choose a single random set $C_{R}$ with MTB index of 283 for further evaluation in the remainder of this paper.

Through simulation, our results show that $C_{M S}^{L F}$ does indeed result in improved tolerable blur performance for the structured light system as compared to $C_{R}$. As shown in the $4^{\text {th }}$ row of Table 1 , for $C_{M S}^{L F}$, the maximum tolerable blur index is 345 , which is a $21.9 \%$ improvement over $C_{R}$.

\section{Decoding with Depth Codes}

In this section, we propose a decoding strategy to further improve virtual DOF in structured light systems with temporally dithered codes. Suppose we numerically blur a focused code set denoted by $C_{F}$ to obtain $C_{b}$, a blurred code set corresponding to defocus distance $b$. If we then decode captured patterns at defocus distance $b$ with respect to $C_{b}$, we would expect decoding to be errorfree. Furthermore, we would expect a range of blur indices over which decoding with respect to $C_{b}$ would still be error-free. In general, it is possible to choose a blurred code set $C_{b}$ that has a tolerable range of blur that overlaps with the tolerable range of $C_{F}$. Consequently, if we decode a captured pattern with respect to both sets, i.e. $C_{F} \cup C_{b}$, we should be able to successfully decode any codeword within the union of the two code sets' tolerable ranges of blur. By using $C_{F}$ in conjunction with $C_{b}$, we effectively increase the virtual DOF of the projector by decoding patterns over a larger range of blur sizes. We refer to this new decoding strategy as depth codes. The key question though is what is the extent to which blurring in $C_{b}$ still results in error free decoding with $C_{F} \cup C_{b}$. Figure 8 shows an example of tolerable range of blurs as a function of blur index for the additional code set used with $C_{F}$. As seen, there is a gradual increase in MTB with the blur index of additional depth code, followed by a sharp decrease. As usual, decoding is accomplished by finding the closest codeword to the captured observation. For depth codes, the position of the codeword in $C_{F}$ or $C_{b}$ is the decoded value. Also, decoding with respect to $C_{F} \cup C_{b}$ requires twice as many distance calculations as either $C_{F}$ or $C_{b}$.

To verify the effectiveness of depth codes, we quantify the increase in the DOF of both $C_{R}$ and $C_{M S}^{L F}$. For $C_{R}$, which is decodable up to blur index 283, we are able to increase the MTB index to 291 when we decode with respect to both the focus code set $C_{R, 0}$ and the set with blur index 273, i.e. $C_{R, 273}$, where $C_{R, N}$ denotes a set generated from $C_{R}$, but blurred with the indicated blur index $N$. This is shown in the third row of Table 1 . This best performance with an additional depth code set is found by evaluating the MTB index when decoding with respect to $C_{F, 0}$ and each increasingly blurred set. We iterate through all blur sizes and choose the set offering the best performance.

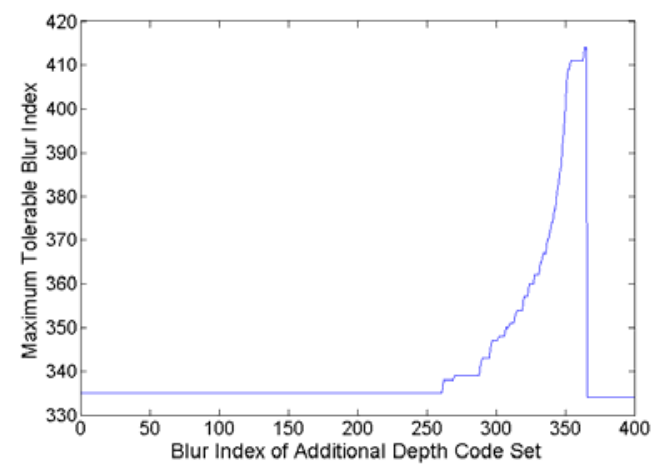

Figure 8: Maximum tolerable blur when decoding with focus code set and one additional blurred set. Results shown for $C_{M S}^{L F}$ of Section 5.3.

We now discuss the depth codes associated with $C_{M S}^{L F}$. The MTB in this case is 416, and is achieved by decoding with $C_{M S}^{L F} \cup C_{M S, 365}^{L F}$, as shown in the $6^{\text {th }}$ row of Table 1. This is a $47.1 \%$ increase over the DOF of $C_{R}$ and $21 \%$ increase over $C_{M S}^{L F}$.

Yet another way to improve the virtual DOF is to introduce deliberate mismatch between the projected codeword $C_{F}$ and decoding codeword $C_{F, M}$. For instance, it is possible to extend the DOF of $C_{M S}^{L F}$ to blur index 386 if the decoding is with respect to $C_{L S, 345}^{H F}$. Specifically, the original code set $C_{M S}^{L F}$ is still projected,

\begin{tabular}{|c|c|c|c|c|c|}
\hline Code Set & $\begin{array}{c}\text { MTB } \\
\text { Index }\end{array}$ & $\begin{array}{c}\text { DOF } \\
\text { @1000mm }\end{array}$ & $\begin{array}{c}\text { DOF @ 1000mm } \\
\text { Improvement } \\
\text { (\%) }\end{array}$ & $\begin{array}{c}\text { DOF } \\
\text { @ } \\
\mathbf{2 5 0 0 m m}\end{array}$ & $\begin{array}{c}\text { DOF @ 2500mm } \\
\text { Improvement } \\
\text { (\%) }\end{array}$ \\
\hline$C_{R}$ & 283 & 49.15 & $0 \%$ & 313.53 & $0.0 \%$ \\
\hline$C_{R, 273}$ & 291 & 50.51 & $2.83 \%$ & 322.47 & \\
\hline$C_{R, 0} \cup C_{R, 273}$ & 291 & 50.51 & $2.83 \%$ & 322.47 & $2.85 \%$ \\
\hline$C_{M S}^{L F}$ & 345 & 59.94 & $21.94 \%$ & 382.95 & $2.85 \%$ \\
\hline$C_{M S, 345}^{L F}$ & 386 & 67.07 & $36.47 \%$ & 429.09 & $36.14 \%$ \\
\hline$C_{M S}^{L F} \cup C_{M S, 365}^{L F}$ & 416 & 72.30 & $47.10 \%$ & 462.99 & $47.67 \%$ \\
\hline
\end{tabular}

Table 1: DOF calculations for system focused at $1000 \mathrm{~mm}$ and $2500 \mathrm{~mm}$. 
only now decoding is performed with respect to $C_{M S, 345}^{L F}$, as shown in the $5^{\text {th }}$ row of Table 1 . Although this method does not provide as much of an improvement as $C_{M S}^{L F^{\prime}} \cup C_{M S, 365}^{L F^{\prime}}$, it is more computationally efficient since we only decode against 1024 rather than 2048 codes.

The intuition behind the above results is as follows: a focus code set can tolerate only so much blur before errors occur. On the other hand, blurred code sets are close to both the original focus codes as well as the more significantly blurred patterns. With codes that are positioned between focused and significantly blurred codes, using a single blurred code set for decoding can actually increase the DOF of the system.

\section{Relating MTB to DOF}

Equation (1) can be used to compute the DOF of our proposed methods by taking into account MTB along with several key parameters of the projector. We assume that the projector has a focal length of $28 \mathrm{~mm}$ and an f-number of 2.5, and present the results for a system focused at $1000 \mathrm{~mm}$ and $2500 \mathrm{~mm}$. For our system parameters, Equation (2) can be approximated as:

$$
d_{D O F}=\frac{2 v N(s-f) r_{b}}{f^{2}}
$$

As seen, a linear increase in tolerable range of blurs leads to a nearly linear increase in the DOF.

Table 1 shows the MTB index, DOF, and percentage improvement of DOF for all the schemes presented in this paper for the projector focused at $1000 \mathrm{~mm}$ and $2500 \mathrm{~mm}$. As seen $C_{M S}^{L F} \cup C_{M S, 365}^{L F}$ depth code results in the largest DOF improvement of about $47 \%$ as compared to single focused decoding of $C_{R}$.

\section{Discussion and Future Work}

We have shown that the DOF performance of a structured light system using temporally dithered codes is highly dependent on the codewords projected by the system. We have shown that by carefully selecting and ordering codes we can improve the performance of a structured light system under blurring.

We have made several assumptions during the codeword design process which in practice might adversely affect the performance of our proposed approach. Firstly, the camera capture has been assumed to be ideal with no image noise, high resolution, and infinite DOF. Additionally, we do not account for ambient lighting, inter-reflections or possible projector lens distortion. To understand the impact of these assumptions, experimental measurements would need to be conducted to determine the virtual DOF improvement in a real system. It would also be interesting to evaluate the performance of error correction codes in conjunction with our proposed approach.

\section{References}

[1] J. Salvi, J. Pagés, and J. Batlle. "Pattern Codification Strategies in Structured Light Systems," Pattern Recognition, vol. 37, no. 4, pp. 827-849, April 2004.

[2] S. Narasimhan, S. Koppal, and S. Yamazaki. "Temporal dithering of illumination for fast active vision," European Conference on Computer Vision, vol. 4, pp. 830-844, October 2008.

[3] S. Zhang, and P. Huang, "High-resolution, real-time three dimensional shape measurement," Optical Engineering, vol. 45, no. 12, 2006.

[4] O. Bimber, and A. Emmerling, "Multifocal Projection: A Multiprojector Technique for Increasing Focal Depth," IEEE Transactions on Visualization and Computer Graphics, vol. 12, vol. 4, pp. 658-667, July 2006.

[5] M. Young, E. Beeson, J. Davis, S. Rusinkiewicz, and R. Ramamoorthi, "Viewpoint-Coded Structured Light," IEEE Conference on Computer Vision and Pattern Recognition, pp.1-8, 2007.

[6] M. Gupta, Y. Tian, S.G. Narasimhan, and Li Zhang, "(De) focusing on global light transport for active scene recovery," IEEE Conference on Computer Vision and Pattern Recognition, pp.2969-2976,2009.

[7] S. Ray, Applied Photographic Optics, second edition. Focal Press, Oxford.

[8] E. R. Dowski, Jr. and W. T. Cathey, "Extended depth of field through wave-front coding," Appl. Opt., vol. 34, pp. 1859-1866, 1995.

[9] A. Levin, R. Fergus, F. Durand, and W.T. Freeman, "Image and depth from a conventional camera with a coded aperture," ACM Trans. Graph, vol. 26, no.3, July 2007.

[10] M. Grosse, and O. Bimber, "Coded aperture projection," ACM SIGGRAPH 2008 Talks (Los Angeles, California, August 11 - 15, 2008). SIGGRAPH '08. ACM, New York, NY, 1-1.

[11] P. Mouroulis, "Depth of field extension with spherical optics," Opt. Express, vol. 16, pp. 12995-13004, 2008.

[12] T. P. Koninckx, A. Griesser, and L.V. Gool, "Realtime range scanning of deformable surfaces by adaptively coded structured light," Proc. 3DIM, pp. 293--300, 2003.

[13] H. Kawasaki, R. Furukawa, R. Sagawa, Y. Yagi, "Dynamic scene shape reconstruction using a single structured light pattern," IEEE Computer Vision and Pattern Recognition, pp. 1-8.

[14] L. Zhang, and S. Nayar, "Projection defocus analysis for scene capture and image display," ACM Trans. Graph, vol.25, no. 3,pp. 907-915, July 2006.

[15] S. Chaudhuri, and A. Rajagopalan, 1999. Depth from defocus: A real aperture imaging approach. SpringerVerlag, New York.

[16] D. Doherty, and G. Hewlett. "10.4: Phased Reset Timing for Improved Digital Micromirror Device (DMD) Brightness," (1998).

[17] S. M. Kay, Fundamentals of statistical signal processing, Detection Theory, vol. 2, Prentice Hall, 1993. 